\title{
Prediction and analysis of HLA-A2/A24-restricted cytotoxic T-lymphocyte epitopes of the tumor antigen MAGE-n using the artificial neural networks method on NetCTL1.2 Server
}

\author{
XIU-MIN ZHANG ${ }^{1 *}$, YANG HUANG $^{2 *}$, ZENG-SHAN LI $^{1}$, HUI LIN $^{1}$ and YAN-FANG SUI ${ }^{1}$ \\ ${ }^{1}$ State Key Laboratory of Cancer Biology, Department of Pathology, and ${ }^{2}$ Department of Emergency \\ Medicine, Xijing Hospital, Fourth Military Medical University, Xi'an, Shaanxi 710032, P.R. China
}

Received July 29, 2010; Accepted September 17, 2010

DOI: $10.3892 / \mathrm{ol} .2010 .193$

\begin{abstract}
Cancer immunotherapy has become one of the most important therapeutic approaches to cancer in the past two decades. Tumor antigen-derived peptides have been widely used to elicit tumor-specific cytotoxic T lymphocytes (CTLs). Antigen-specific CTLs induced by MAGE-derived peptides have proven to be highly efficacious in the prevention and treatment of various types of tumor. MAGE-n is a new member of the MAGE gene family and has been shown to be closely associated with hepatocellular carcinoma. It is highly homologous to the MAGE-A gene subfamily, particularly to MAGE-3 (93\%). MAGE-n-derived peptide QLVFGIEVV is a novel HLA-A2.1-restricted CTL epitope that induces MAGE$\mathrm{n}$-specific CTLs in vitro. Identification of these CTL epitopes may lead to clinical applications of these peptides as cancer vaccines for patients with MAGE- $n^{+} / \mathrm{HLA}-\mathrm{A} 2^{+}$tumors. In the present study, HLA-A/A24-restricted CTL epitopes of antigen MAGE-n were predicted using the NetCTL1.2 Server on the web, COMB $>0.85$. The results showed that the NetCTL1.2 Server prediction method improved prediction efficacy and accuracy. Additionally, 8 HLA-A2- and 9 HLA-A24-restricted CTL epitope candidates (nonamers) derived from the tumor antigen MAGE-n were predicted. These nonamers, following identification via experimentation, may contribute to the development of potential antigen peptide tumor vaccines.
\end{abstract}

\section{Introduction}

Recent studies have shown that tumor antigens, particularly tumor-specific antigens, which induce tumor-specific cytotoxic

Correspondence to: Dr Yan-Fang Sui, State Key Laboratory of Cancer Biology, Department of Pathology, Xijing Hospital, Fourth Military Medical University, Xi'an, Shaanxi 710032, P.R. China

E-mail: suiyanf@gmail.com

${ }^{*}$ Contributed equally

Key words: MAGE-n, HLA-A2/A24, cytotoxic T-lymphocyte epitope, prediction
T lymphocytes (CTLs) and damage tumor cells, are a significant component of tumor vaccines. Epitope peptide vaccine has been of particular interest as it induces specific CTL in vitro and in vivo in order to kill target cells. Previously, a number of human genes that code for tumor antigens identified by autologous CTLs were isolated $(1,2)$, and the epitopes derived from these tumor antigens were further identified to serve as targets for CTLs in the context of HLA class I molecules (3). MAGE-n is a newly identified member of the MAGE gene family which was first reported by our laboratory (Genebank, locus no. AF443295) (4), and was also found to be highly homologous to MAGE-A subfamily genes. Since HLA-A2/ A24 is one of the most frequently expressed molecules in the Chinese population $(5,6)$, it is crucial to identify the tumor antigen epitopes which are presented by HLA-A2/A24 and to induce epitope-specific CTLs against tumor cells. The present study reported a simple and efficient bioinformatics method to identify candidate HLA-A2/A24-restricted CTL epitopes from the tumor antigen MAGE-n.

\section{Materials and methods}

MAGE-n (316 aa) was selected as the antigen of interest in this study. The amino acid sequences of the tumor antigen were obtained from the Genbank database: MSLEQRSQHCKPEEGLEARGEALGLVGAQAPATEE QEAASSSSTLVEVTLGEVPAAESPDPPQSPQGASSLP TTMNYPLWSQSYEDSSNQEEEGPSTFPDLESEFQ AALSRKVAELVHFLLLKYRAREPFTKAEMLGS VIR NFQDFFPVIFSEASEYLQLVFGIEV VEV V RIGHLYILVTCLGLSYDGLLGDNQIMPKTGFLIIVLV MIA MEGGHA PEEEIWEELSVMEVYDGREHSAY GEPRKLLTQDLVQEK Y LEY RQVPDSDPARYEFL WGPRALAETSYVK VLEYVIKVSARVRFFFPSLREAA LREEEEGV.

Methods. HLA-A/A24-restricted CTL epitopes of antigen MAGE-n were predicted using the NetCTL1.2 Server on the web. The NetCTL1.2 Server (http://www.cbs.dtu.dk/services/ NetCTL) was used for CTL epitope prediction according to the following steps: i) input (paste) the MAGE-n protein sequence in the 'Submission' window, ii) select A2/A24 (HLA-A2/ 


\section{SUBMISSION}

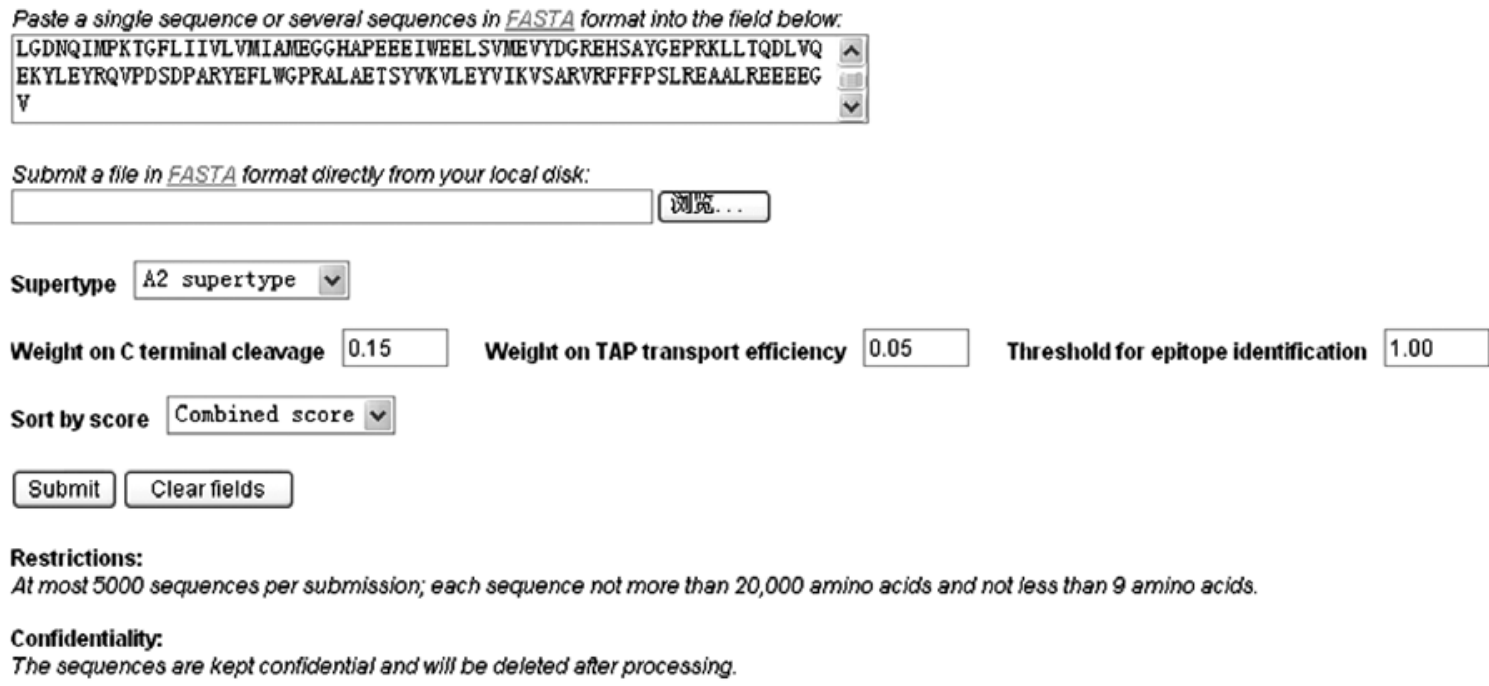

Figure 1. The web page of the NetCTL-1.2 Server epitope prediction.

Table I. HLA-A2-restricted epitope prediction results derived from MAGE-n.

\begin{tabular}{rlccccc}
\hline ID & Sequence pep & aff & aff_rescale & cle & tap & COMB \\
\hline 201 & FLIIVLVMI & 0.6883 & 1.0260 & 0.9554 & 0.4980 & 1.1942 \\
176 & YILVTCLGL & 0.6209 & 0.9255 & 0.9451 & 1.1290 & 1.1237 \\
289 & YVIKVSARV & 0.6177 & 0.9208 & 0.9484 & 0.3350 & 1.0798 \\
108 & ALSRKVAEL & 0.5660 & 0.8437 & 0.9285 & 1.2680 & 1.0464 \\
174 & HLYILVTCL & 0.5486 & 0.8178 & 0.9762 & 1.0400 & 1.0163 \\
158 & LQLVFGIEV & 0.5876 & 0.8760 & 0.4210 & 0.3600 & 0.9571 \\
276 & RALAETSYV & 0.5436 & 0.8103 & 0.1751 & 0.4840 & 0.8608 \\
24 & GLVGAQAPA & 0.5000 & 0.7453 & 0.9252 & -0.5690 & 0.8556
\end{tabular}

ID, protein identifier; sequence pep, peptide sequence; aff, predicted MHC binding affinity; aff_rescale, rescale binding affinity; cle, $\mathrm{C}$ terminal cleavage affinity; tap, TAP transport efficiency; COMB, prediction score.

Table II. HLA-A24-restricted epitope prediction results derived from MAGE-n.

\begin{tabular}{|c|c|c|c|c|c|c|}
\hline ID & Sequence pep & aff & aff_rescale & cle & tap & COMB \\
\hline 156 & EYLQLVFGI & 0.7953 & 1.6935 & 0.2612 & 0.6670 & 1.7661 \\
\hline 195 & IMPKTGFLI & 0.6701 & 1.4269 & 0.0687 & 0.6150 & 1.4679 \\
\hline 293 & VSARVRFFF & 0.5493 & 1.1698 & 0.1103 & 2.7550 & 1.3240 \\
\hline 97 & TFPDLESEF & 0.4816 & 1.0254 & 0.9542 & 2.7200 & 1.3045 \\
\hline 76 & NYPLWSQSY & 0.3996 & 0.8510 & 0.9783 & 3.2470 & 1.1601 \\
\hline 142 & NFQDFFPVI & 0.4356 & 0.9276 & 0.7956 & 0.8390 & 1.0889 \\
\hline 138 & SVIRNFQDF & 0.3807 & 0.8106 & 0.5758 & 2.7650 & 1.0353 \\
\hline 282 & SYVKVLEYV & 0.4190 & 0.8923 & 0.4156 & 0.7200 & 0.9906 \\
\hline 113 & VAELVHFLL & 0.3159 & 0.6727 & 0.9570 & 1.0260 & 0.8676 \\
\hline
\end{tabular}

ID, protein identifier; sequence pep, peptide sequence; aff, predicted MHC binding affinity; aff_rescale, rescale binding affinity; cle, $\mathrm{C}$ terminal cleavage affinity; tap, TAP transport efficiency; COMB, prediction score. 
A24) from the 'Supertype' menu, iii) enter $0.15,0.05,1.0$ in the 'Weight on $\mathrm{C}$ terminal cleavage', 'Weight on TAP transport efficiency' and 'Threshold for epitope identification' windows, respectively, iv) select Combined score from the dropdown menu of 'Sort by score', and v) Submit to run the remote forecast. The predicted value includes the ID (protein identifier), sequence pep (peptide sequence), aff (predicted MHC binding affinity), aff_rescale (rescale binding affinity), cle (C terminal cleavage affinity), tap (TAP transport efficiency) and COMB (prediction score). According to the characteristic property, the greater the COMB, the higher the specificity of the predicted result; 0.85 was selected as the threshold. Finally, the candidate HLA-A2/A24-restricted CTL epitope peptides were obtained by excluding the peptides already confirmed from the prediction results.

\section{Results}

The NetCTL-1.2 prediction values of all possible nonamers of a given protein sequence were added together. The peptides of each protein were selected for further analysis. The primary predicted epitopes were compared to those of previous studies (7-9). It was found that the 4 reported HLA-A2-restricted CTL epitopes, i.e., MAGE-1 ${ }_{278-286}(\mathrm{KVLEYVIKV),} \mathrm{MAGE-}$ $3_{278-286}(\mathrm{KVAELVHFL}), \quad$ AGE-3 ${ }_{271-279}(\mathrm{FLWGPRALV})$ and MAGE- $\mathrm{n}_{159-167}(\mathrm{QLVFGIEVV})$ were eliminated from the NetCTL-1.2 prediction results. The epitopes with COMB scores $<0.85$ were eliminated from the prediction results. A total of 8 HLA-A2- and 9 HLA-A24-restricted CTL epitope candidates (nonamers) derived from the tumor antigen MAGE-n were predicted. The results of predicted epitopes are shown in Tables I and II, i.e., that the NetCTL1.2 Server prediction method improved prediction efficacy and accuracy.

\section{Discussion}

Recently, with the innovation of immunology methodology, the molecular mechanisms of tumor antigen presentation and $\mathrm{CD}^{+} \mathrm{T}$-cell activation have been gradually elucidated. Tumor antigens and their coding genes have been identified in a consecutive manner. The breakthrough in theoretical tumor immunity has accelerated the development of vaccines targeting tumor antigens. The induction of antigenspecific CTLs has been suggested to be highly efficacious in the prevention and treatment of various types of tumor. Induction of potent anti-tumor CTL responses results in the regression and prevention of metastasis formation, as shown in experimental model tumor systems $(10,11)$. Thus, efforts towards the development of cancer immunotherapy have recently focused on the generation of tumor-specific T-cell responses.

Epitope prediction was recently conducted in tumor antigen specificity for CTL epitope identification in a majority of studies. Approximately 120 CTL epitopes presented by HLA-A, B and C molecules were reported (12), and various epitopes were used as peptide vaccines in animal and clinical experiments $(13,14)$. Schirle et al reported that two new CTL epitopes of gastrointestinal tumors were identified by epitope prediction combined with acid elution methods (15).
Dong et al found that MAGE-n-derived peptide QLVFGIEVV was a new HLA-A2.1-restricted CTL epitope that induces MAGE-n-specific CTLs in vitro (9). Identification of these CTL epitopes allows for clinical applications of these peptides as cancer vaccines for patients with MAGE-n ${ }^{+} / \mathrm{HLA}-\mathrm{A} 2^{+}$ tumors. Our previous study showed that the combination of MAGE-3 and MAGE-n epitopes induced more effective antitumor immune responses than either of the peptides alone (16). In addition, the peptide-specific activity was observed to be in a MHC-restricted manner. This study suggested that the combination of a number of tumor antigen-derived peptides is more efficacious as a peptide-based cancer immunotherapy.

The combination of epitope prediction, epitope reconstruction and immunological methods improves the efficacy and accuracy of CTL epitope studies (9). The identification of human tumor-rejection antigens and CTL epitopes of these antigens allows for the development of new cancer vaccines since patients are likely to benefit from immunization with an identified CTL epitope. Among the identified tumor-rejection antigens, MAGE gene products are of particular interest due to their wide expression in various types of tumor and their potential to induce tumor-specific CTL responses. A previous study showed that a vaccine with 5 common HLA-I-restricted epitopes was beneficial in $80-90 \%$ of patients (17). It was also found that 8-9 common HLA-I-restricted epitopes may exhibit benefical effects on patients worldwide (17). The identification of the HLA-A2-restricted CTL epitopes from melanoma antigen MAGE-n is crucial. In the present study, the HLA-A2/ A24-restricted CTL epitopes of MAGE-n were predicted using the methods from the NetCTL1.2 Server. These candidate epitopes were selected for further immunology experiments. The results showed that the NetCTL1.2 Server prediction method improves prediction efficacy and accuracy. These nonamers, following identification through experimentation may contribute to the development of potential antigen peptide tumor vaccines.

\section{Acknowledgements}

This study was supported by grants from the China National Natural Science Foundation (no. 30901735) and the Science and Technology Project of Shaanxi Province, China [no. 2007K09-05(2)].

\section{References}

1. Katsura F, Eura M, Chikamatsu K, Oiso M, Yumoto E and Ishikawa T: Analysis of individual specific cytotoxic T lymphocytes for two MAGE-3-derived epitopes presented by HLA-A24. Jpn J Clin Oncol 30: 117-121, 2000.

2. Boel P, Wildmann C, Sensi ML, Brasseur R, Renauld JC, Coulie P, Boon T and van der Bruggen P: BAGE: a new gene encoding an antigen recognized on human melanomas by cytolytic T lymphocytes. Immunity 2: 167-175, 1995.

3. Renkvist N, Castelli C, Robbins PF and Parmiani G: A listing of human tumor antigens recognized by $\mathrm{T}$ cells. Cancer Immunol Immunother 50: 3-15, 2001.

4. Wu W, Sui YF, Ye J, Li ZS, Guo AL, Yang XW, QU P and Zhang XM: The cloning of tumor-associated gene MAGE in human hepatocellular carcinoma cell line. Chin J Cell Mol Immunol 18: 270-274, 2002.

5. Ming LH, Zhu YR, Harris CC, Wang XH, Wu ZY and Sun ZT: Association of HLA-A02 genotype with hepatocellular carcinoma in a prevalent area of China. Chin J Immunol 15: 305-310, 1999. 
6. Cai SL, Zhao HT, Leng XS, Cheng TH, Gong SY, Peng JR, Cong X, Wang Y, Rui JA, Hui YZ, Du RY and Chen WF: Melanoma antigen-3 expression in human hepatocellular carcinoma. Chin J Surg 38: 693-696, 2000.

7. Pascolo S, Schirle M, Guckel B, Dumrese T, Stumm S, Kayser S, Moris A, Wallwiener D, Rammensee HG and Stevanovic S: A MAGE-A1 HLA-A*0201 epitope identified by mass spectrometry. Cancer Res 61: 4027-4077, 2001.

8. Vander Bruggen P, Bastin J, Boon T, Gajewski T, Coulie PG, Boël P, De Smet C, Traversari C, Townsend A and Boon T: A peptide encoded by human gene MAGE-3 and presented by HLA-A2 induces cytolytic T lymphocytes that recognize tumor cells expressing MAGE-3. Eur J Immunol 24: 3038-3043, 1994.

9. Dong HL, Li ZS, Ye J, Qu P, Huang YY, Wu W, Lu SY, Chen GS and Sui YF: Identification of HLA-A2-restricted CTL epitope encoded by the MAGE-n gene of human hepatocellular carcinoma. Cancer Biol Ther 3: 891-898, 2004.

10. Marchand M, van Baren N, Weynants P, et al: Tumor regression observed in patients with metastic melanoma treated with an antigenic peptide encoded by gene MAGE- 3 and presented by HLA-A1. Int J Cancer 80: 219-230, 1999.

11. Horiguchi Y, Nukaya I, Okazawa K, Kawashima I, Fikes J, Sette A, Tachibana M, Takesako K and Murai M: Screening of HLA-A24-restricted epitope peptides from prostate-specific membrane antigen that induce specific antitumor cytotoxic T lymphocytes. Clin Cancer Res 8: 3885-3892, 2002.
12. Feuerer M, Beckhove P, Bai L, Solomayer EF, Bastert G, Diel IJ, Pedain C, Oberniedermayr M, Schirrmacher V and Umansky V: Therapy of human tumors in NOD/SCID mice with patientderived reactivated memory $\mathrm{T}$ cells from bone marrow. Nat Med 7: 452-458, 2001

13. Dagher R, Long LM, Read EJ, Leitman SF, Carter CS, Tsokos M, Goletz TJ, Avila N, Berzofsky JA, Helman LJ and Mackall CL: Pilot trial of tumor-specific peptide vaccination and continuous infusion interleukin-2 in patients with recurrent Ewing sarcoma and alveolar rhabdomyosarcoma: an inter-institute NIH study. Med Pediatr Oncol 38: 158-164, 2002.

14. Schiavetti F, Thonnard J, Colau D, Boon T and Coulie PG: A human endogenous retroviral sequence encoding an antigen recognized on melanoma by cytolytic $\mathrm{T}$ lymphocytes. Cancer Res 62: 5510-5516, 2002.

15. Schirle M, Keilholz W, Weber B, Gouttefangeas C, Dumrese T, Becker HD, Stevanović S and Rammensee HG: Identification of tumor-associated MHC class I ligands by a novel T cellindependent approach. Eur J Immunol 30: 2216-2225, 2000.

16. Zhang XM, Zhang YF, Huang Y, Qu P, Ma B, Si SY, Li ZS, Li WX, Li X, Ge W, Hu PZ and Sui YF: The anti-tumor immune response induced by a combination of MAGE-3/MAGE-nderived peptides. Oncol Rep 20: 245-252, 2008.

17. Sidney J, Grey HM, Kubo RT and Sette A: Practical, biochemical and evolutionary implications of the discovery of HLA class I supermotifs. Immunol Today 17: 261-266, 1996. 